\title{
Gross Total Resection of a Recurrent in-and-around Cavernous Sinus Meningioma through a Combined Transcavernous Anterior and Middle Infratemporal Fossa Approach with Extracranial-Intracranial Bypass
}

Yoichi Nonaka ${ }^{1}$ Naokazu Hayashi ${ }^{1}$ Takanori Fukushima ${ }^{2,3}$

${ }^{1}$ Department of Neurosurgery, Tokai University School of Medicine, Kanagawa, Japan

2 Division of Neurosurgery, Duke University Medical Center, Durham, Address for correspondence Yoichi Nonaka, MD, PhD, 143 North Carolina, United States

${ }^{3}$ Carolina Neuroscience Institute, Raleigh, North Carolina, United States Shimokasuya, Isehara, Kanagawa 259-1193, Japan (e-mail: ynonaka1971@yahoo.co.jp).

J Neurol Surg B Skull Base 2022;83(suppl S3):e608-e609.

\author{
Abstract \\ Keywords \\ - cavernous sinus \\ - cerebral bypass \\ - recurrent \\ meningioma \\ - skull base surgery \\ - transcavernous \\ approach
}

Funding

None.

Conflict of Interest

None declared.

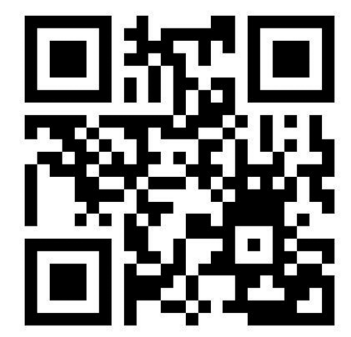

received

April 1, 2020

accepted

October 27, 2020

published online

March 18, 2021
Objectives The study aims to describe surgical management of an invasive cavernous sinus meningioma with a combination of several skull base approaches and bypass surgery.

Design This study is an operative video.

Results Resection of the recurrent skull base meningioma is still challenging, especially if the tumor involves or encases the carotid artery. Cerebral bypass surgery is an essential adjunct in the armamentarium of skull base surgery when vessel reconstruction is required. In this paper, we describe our experience of successful treatment of an invasive recurrent skull base meningioma, which involved the entire cavernous sinus and the internal carotid artery. A 46-year-old woman presented with a 2-year history of gradually worsening left-sided exophthalmos and visual impairment. The patient had previously undergone two craniotomies for resection of the left-sided spheno-orbital meningioma. Pathological diagnosis was chordoid meningioma, which www.thieme.com/skullbasevideos

www.thieme.com/jnlsbvideos
Dol https://doi.org/ $10.1055 / \mathrm{s}-0041-1725935$ ISSN 2193-6331.

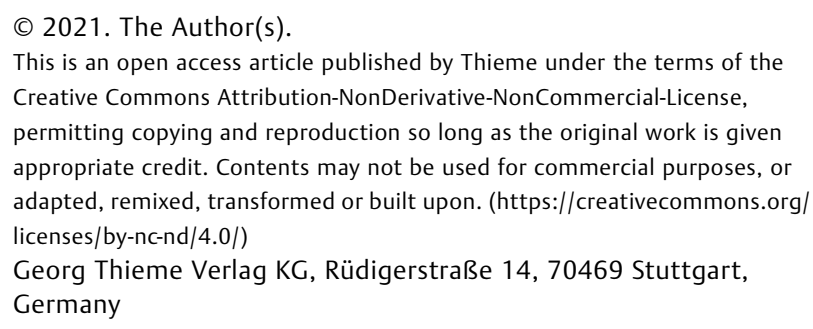
permitting copying and reproduction so long as the original work is given appropriate credit. Contents may not be used for commercial purposes, or adapted, remixed, transformed or built upon. (https://creativecommons.org/ licenses/by-nc-nd/4.0/) Georg Thieme Verlag KG, Rüdigerstraße 14, 70469 Stuttgart, Germany 
is classified as an intermediate-grade meningioma. The second surgery had been performed for a rapid tumor regrowth 6 months after the first surgery. The patient lost her left-side vision after the second surgery. Aggressive tumor regrowth was confirmed with extension into the left orbit, infratemporal fossa, and cavernous sinus with engulfment of the carotid artery. A balloon occlusion test revealed intolerance of the left internal carotid artery occlusion. Considering the patient's age, tumor behavior, and intolerance of the carotid artery of the lesion side, we scheduled gross total resection of the tumor with vessel reconstruction.

Conclusion Although cerebral bypass surgery is a technically challenging procedure, it plays an important role in the surgical management of the complex vessel-engulfing tumor. The link to the video can be found at https://youtu.be/GCmpxK3hW18.

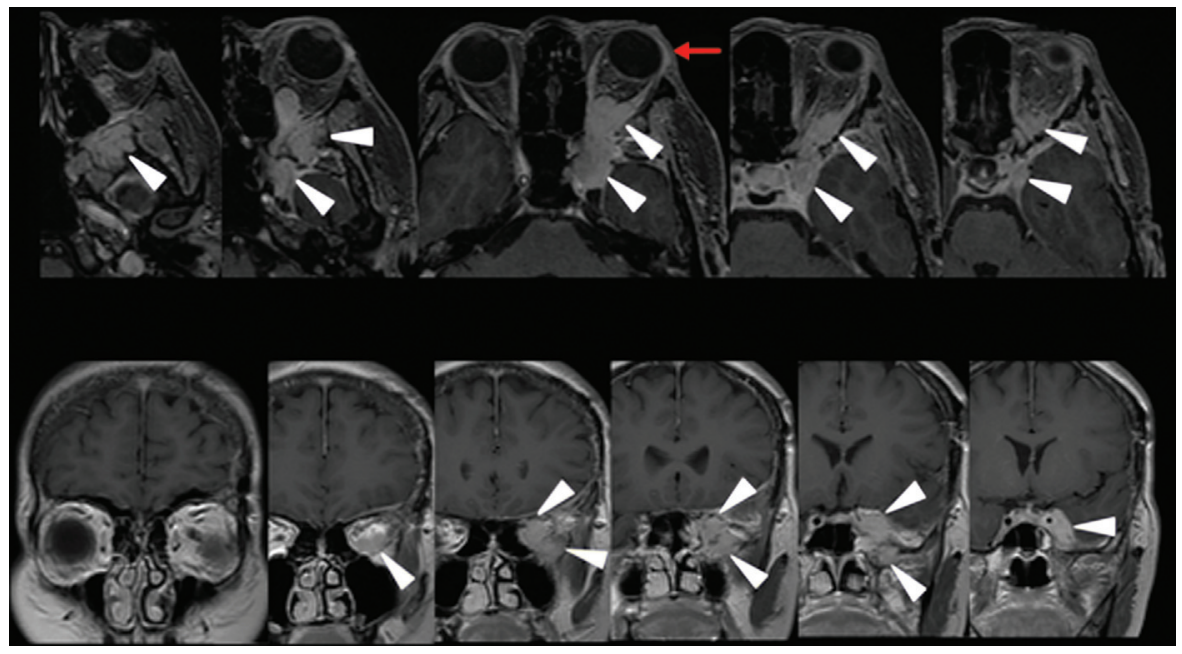

Fig. 1 Preoperative postcontrast magnetic resonance images (upper; axial, lower; coronal) demonstrate tumor extension into the left orbit, infratemporal fossa, and cavernous sinus (white arrowheads). An axial image shows exophthalmos on the left side (arrowhead).

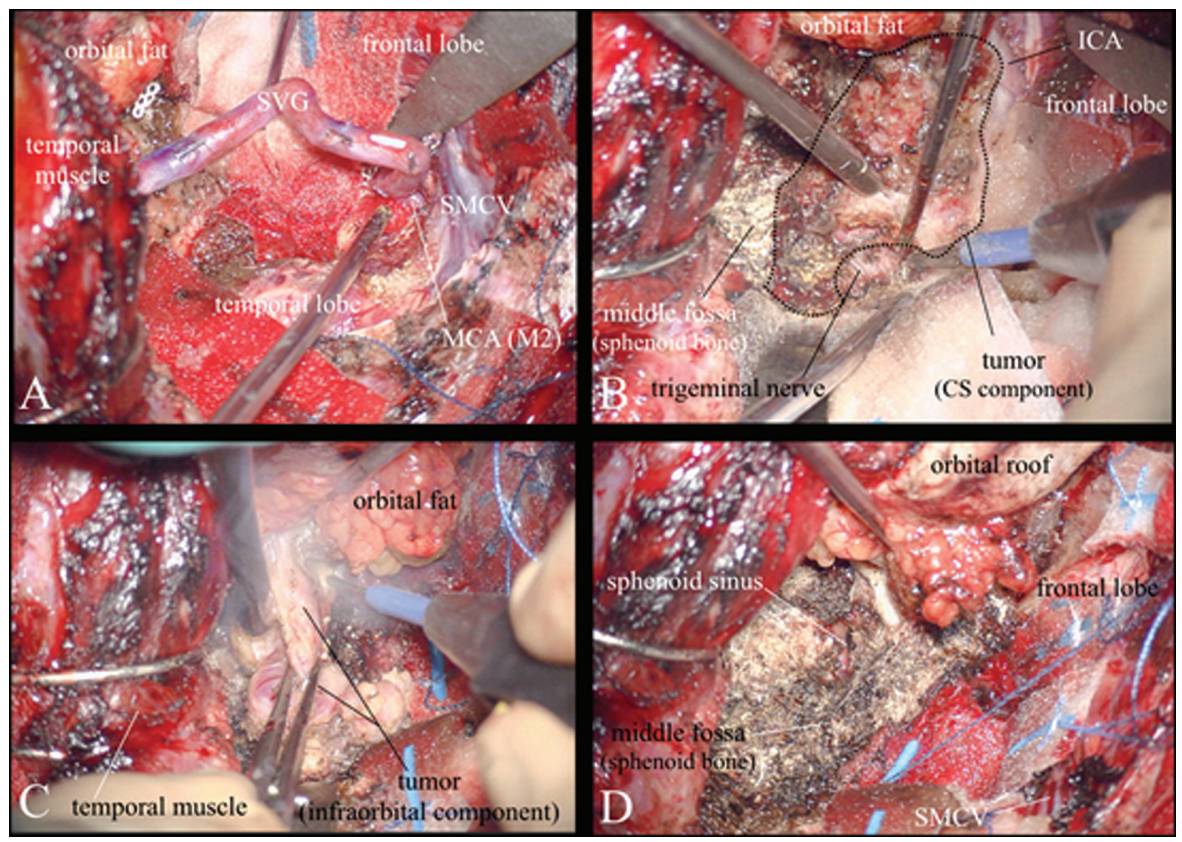

Fig. 2 Intraoperative still images. (A) High flow EC-IC bypass is performed before tumor removal. A saphenous vein graft is anastomosed to the left M2 segment of the middle cerebral artery. The tip of the left temporal lobe is partially removed. (B) The cavernous sinus component of the tumor is elevating from the sphenoid bone of the middle fossa by monopolar cautery. (C) The inferior part of the orbital component of tumor is resected. (D) Final view of the gross total resection of the tumor. The sphenoid bone of the parasellar middle fossa part is exposed. CS, cavernous sinus; EC-IC bypass, extracranial-intracranial bypass; ICA, internal carotid artery; SMCV, superficial middle cerebral vein. 